To appear in Euro. Jnl of Applied Mathematics

\title{
Direct construction method for conservation laws of partial differential equations. Part II: General treatment
}

\author{
STEPHEN C. ANCO ${ }^{1}$ and GEORGE BLUMAN ${ }^{2}$ \\ ${ }^{1}$ Department of Mathematics, Brock University, St. Catharines, ON Canada L2S 3A1 \\ email: sanco@brocku.ca \\ ${ }^{2}$ Department of Mathematics, University of British Columbia, Vancouver, BC Canada V6T 1Z2 \\ email: bluman@math.ubc.ca
}

This paper gives a general treatment and proof of the direct conservation law method presented in Part I (see [3]). In particular, the treatment here applies to finding the local conservation laws of any system of one or more partial differential equations expressed in a standard Cauchy-Kovalevskaya form. A summary of the general method and its effective computational implementation is also given.

\section{Introduction}

In this paper we present a general treatment of the direct conservation law method introduced in Part I (see Ref. [3]). In particular, in Sec. 2 we show how to find the local conservation laws for any system of one or more PDEs expressed in a standard CauchyKovalevskaya form. We specifically treat $n$th order scalar PDEs in Sec. 3. In Sec. 固 we summarize the general method and discuss its effective implementation in computational terms.

In order to make the treatment uniform, it is convenient to work with Cauchy-Kovalevskaya systems of PDEs as follows.

Definition 1.1 A PDE system with any number of independent and dependent variables has Cauchy-Kovalevskaya form in terms of a given independent variable if the system is in solved form for a pure derivative of the dependent variables with respect to the given independent variable, and if all other derivatives of dependent variables in the system are of lower order with respect to that independent variable.

Typically, scalar PDEs admit a Cauchy-Kovalevskaya form by singling out a derivative with respect to one independent variable, or by making a point transformation (more generally a contact transformation) on the independent variables. For example: the wave equation

$$
u_{t x}=0
$$

admits the Cauchy-Kovalevskaya form $u_{t t}=u_{x x}$ after the point transformation $t \rightarrow t-x$, 
$x \rightarrow x+t$; the harmonic equation

$$
u_{x x}+u_{y y}=0
$$

admits the Cauchy-Kovalevskaya form $u_{y y}=-u_{x x}$ with respect to $y$. A less trivial example is the Kadomtsev-Petviashvili equation [10]

$$
u_{t x}+\left(u u_{x}\right)_{x}+u_{x x x x} \pm u_{y y}=0 .
$$

This equation admits two obvious Cauchy-Kovalevskaya forms: $u_{y y}=\mp\left(u_{t x}+\left(u u_{x}\right)_{x}+\right.$ $\left.u_{x x x x}\right)$ which is a second-order PDE with respect to $y$; and $u_{x x x x}=\mp u_{y y}-u_{t x}-\left(u u_{x}\right)_{x}$ which is a fourth-order PDE with respect to $x$.

As examples which are more involved, consider the modified Benjamin-Bona-Mahoney equation [4]

$$
u_{t}+\left(1+u^{2}\right) u_{x}-u_{x x t}=0,
$$

and the symmetric regularized long wave equation [12]

$$
u_{t t}+u_{x x}+u u_{t x}+u_{x} u_{t}+u_{t t x x}=0 .
$$

As it stands the modified Benjamin-Bona-Mahoney equation is not of Cauchy-Kovalevskaya form with respect to either $t$ or $x$, since the $t$ derivatives of $u$ appear in both pure and mixed derivative terms, while the highest order $x$ derivative of $u$ appears in a mixed derivative involving $t$ and hence is not in solved form. Nevertheless, if one makes the point transformation $t \rightarrow t, x \rightarrow x-t$, then the modified Benjamin-Bona-Mahoney equation becomes $u_{x x x}-u_{x x t}+u^{2} u_{x}+u_{t}=0$ which now is of third-order Cauchy-Kovalevskaya form with respect to $x$. The situation for the symmetric regularized long wave equation is similar. It is not of Cauchy-Kovalevskaya form as it stands, but after one makes the point transformation $t \rightarrow t-x, x \rightarrow x+t$ it is of fourth-order Cauchy-Kovalevskaya form with respect to $t$ or $x: u_{t t t t}+u_{x x x x}-2 u_{t t x x}+(2-u) u_{t t}+(2+u) u_{x x}+u_{t}{ }^{2}-u_{x}{ }^{2}=0$.

Many PDE systems can be handled similarly to scalar PDEs. For example, the vector nonlinear Schroedinger equation

$$
i \vec{u}_{t}+\vec{u}_{x x} \pm f(|\vec{u}|) \vec{u}=0, \quad \vec{u}=\left(u^{1}, \ldots, u^{n}\right)
$$

admits the first-order Cauchy-Kovalevskaya form $\vec{u}_{t}=i \vec{u}_{x x} \pm i f(|\vec{u}|) \vec{u}$ with respect to $t$, as well as the second-order Cauchy-Kovalevskaya form $\vec{u}_{x x}=-i \vec{u}_{t} \mp f(|\vec{u}|) \vec{u}$ with respect to $x$. A less obvious example is Navier's equations of isotropic elasticity,

$$
\begin{aligned}
\kappa u_{x x}+\mu u_{y y}+(\kappa-\mu) v_{x y} & =0, \\
(\kappa-\mu) u_{x y}+\mu v_{x x}+\kappa v_{y y} & =0,
\end{aligned}
$$

$\kappa=$ const, $\mu=$ const. This PDE system admits a second-order Cauchy-Kovalevskaya form with respect to $x$ or $y: u_{x x}=-\frac{\mu}{\kappa} u_{y y}+\left(\frac{\mu}{\kappa}-1\right) v_{x y}$ and $v_{x x}=-\frac{\kappa}{\mu} v_{y y}+\left(1-\frac{\kappa}{\mu}\right) u_{x y}$.

In general any Cauchy-Kovalevskaya form of a system of one or more PDEs can be used with no loss of completeness in finding the conservation laws admitted by the system. Given a Cauchy-Kovalevskaya PDE system, we let $t$ denote the independent variable in the derivative which appears in solved form in the PDEs, with the remaining independent variables denoted by $\boldsymbol{x}=\left(x^{1}, \ldots, x^{n}\right)$. In order to obtain the most effective formulation of 
the direct conservation law method, it is convenient to express the system in its equivalent first-order (evolution) form with respect to $t$.

Hence, we consider a first-order Cauchy-Kovalevskaya system of PDEs with $N$ dependent variables $\boldsymbol{u}=\left(u^{1}, \ldots, u^{N}\right)$ and $n+1$ independent variables $(t, \boldsymbol{x})$,

$$
G^{\sigma}=\frac{\partial u^{\sigma}}{\partial t}+g^{\sigma}\left(t, \boldsymbol{x}, \boldsymbol{u}, \partial_{\boldsymbol{x}} \boldsymbol{u}, \ldots, \partial_{\boldsymbol{x}}^{m} \boldsymbol{u}\right)=0, \sigma=1, \ldots, N
$$

with $\boldsymbol{x}$ derivatives of $\boldsymbol{u}$ up to some order $m$. We use $\partial_{\boldsymbol{x}} \boldsymbol{u}, \partial_{\boldsymbol{x}}^{2} \boldsymbol{u}$, etc. to denote all derivatives of $u^{\sigma}$ of a given order with respect to $x^{i}$. We denote partial derivatives $\partial / \partial t$ and $\partial / \partial x^{i}$ by subscripts $t$ and $i$ respectively. Corresponding total derivatives are denoted by $D_{t}$ and $D_{i}$. We let $\left(\mathcal{L}_{g}\right)_{\rho}^{\sigma}$ denote the linearization operator of $g^{\sigma}$ defined by

$$
\left(\mathcal{L}_{g}\right)_{\rho}^{\sigma} V^{\rho}=\frac{\partial g^{\sigma}}{\partial u^{\rho}} V^{\rho}+\frac{\partial g^{\sigma}}{\partial u_{i}^{\rho}} D_{i} V^{\rho}+\cdots+\frac{\partial g^{\sigma}}{\partial u_{i_{1} \cdots i_{m}}^{\rho}} D_{i_{1} \cdots i_{m}} V^{\rho},
$$

and we let $\left(\mathcal{L}_{g}^{*}\right)_{\rho}^{\sigma}$ denote the adjoint operator defined by

$$
\left(\mathcal{L}_{g}^{*}\right)_{\rho}^{\sigma} W_{\sigma}=\frac{\partial g^{\sigma}}{\partial u^{\rho}} W_{\sigma}-D_{i}\left(\frac{\partial g^{\sigma}}{\partial u_{i}^{\rho}} W_{\sigma}\right)+\cdots+(-1)^{m} D_{i_{1} \cdots i_{m}}\left(\frac{\partial g^{\sigma}}{\partial u_{i_{1} \cdots i_{m}}^{\rho}} W_{\sigma}\right)
$$

acting on arbitrary functions $V^{\rho}, W_{\sigma}$ respectively.

Throughout we use the summation convention for repeated lower-case indices; we use an explicit summation sign where needed for summing over non-indices.

\section{General treatment}

We start by considering the determining equations for symmetries and adjoint symmetries. Suppose $\mathrm{X}$ is the infinitesimal generator of a symmetry leaving invariant PDE system (1.1). We denote $\mathrm{X} u^{\sigma}=\eta^{\sigma}$, which satisfies

$$
0=D_{t} \eta^{\sigma}+\left(\mathcal{L}_{g}\right)_{\rho}^{\sigma} \eta^{\rho}, \sigma=1, \ldots, N
$$

for all solutions $\boldsymbol{u}(t, \boldsymbol{x})$ of Eq. (1.1). This linearization of Eq. (1.1) is the determining equation for symmetries (point-type as well as first-order and higher-order type [1]) $\eta^{\sigma}\left(t, \boldsymbol{x}, \boldsymbol{u}, \partial \boldsymbol{u}, \ldots, \partial^{p} \boldsymbol{u}\right)$ of the PDE system (1.1), where $\partial^{j} \boldsymbol{u}$ denotes all $j$ th order derivatives of $\boldsymbol{u}$ with respect to all independent variables $t, \boldsymbol{x}$. The adjoint of Eq. (2.1) is given by

$$
0=-D_{t} \omega_{\sigma}+\left(\mathcal{L}_{g}^{*}\right)_{\sigma}^{\rho} \omega_{\rho}, \sigma=1, \ldots, N
$$

which is the determining equation for adjoint symmetries $\omega_{\sigma}\left(t, \boldsymbol{x}, \boldsymbol{u}, \partial \boldsymbol{u}, \ldots, \partial^{p} \boldsymbol{u}\right)$ of the PDE system (1.1). In general, solutions of the adjoint symmetry equation (2.2) are not solutions of the symmetry equation (2.1), and there is no interpretation of adjoint symmetries in terms of an infinitesimal generator leaving anything invariant.

In order to solve the determining equations for $\eta^{\sigma}$ and $\omega_{\sigma}$, one works on the space of solutions of the PDE system. This means we use the PDEs to eliminate $u_{t}^{\sigma}$ in terms of $u^{\sigma}, u_{i}^{\sigma}$, etc. In particular, without loss of generality, we are free to let $\eta^{\sigma}$ and $\omega_{\sigma}$ have 
no dependence on $u_{t}^{\sigma}$ and its differential consequences. Let

$$
\mathcal{D}_{t}=\partial_{t}-\left(g^{\rho} \partial_{u^{\rho}}+\left(D_{i} g^{\rho}\right) \partial_{u_{i}^{\rho}}+\cdots\right)
$$

which is the total derivative with respect to $t$ on the solution space of PDE system (1.1). (In particular, $D_{t}=\mathcal{D}_{t}$ when acting on all solutions $\boldsymbol{u}(t, \boldsymbol{x})$.) Then the determining equations explicitly become

$$
\begin{aligned}
0= & \mathcal{D}_{t} \eta^{\sigma}+\left(\mathcal{L}_{g}\right)_{\rho}^{\sigma} \eta^{\rho} \\
= & \frac{\partial \eta^{\sigma}}{\partial t}-\left(\frac{\partial \eta^{\sigma}}{\partial u^{\rho}} g^{\rho}+\frac{\partial \eta^{\sigma}}{\partial u_{i}^{\rho}} D_{i} g^{\rho}+\cdots+\frac{\partial \eta^{\sigma}}{\partial u_{i_{1} \cdots i_{p}}^{\rho}} D_{i_{1}} \cdots D_{i_{p}} g^{\rho}\right) \\
& \quad+\frac{\partial g^{\sigma}}{\partial u^{\rho}} \eta^{\rho}+\frac{\partial g^{\sigma}}{\partial u_{i}^{\rho}} D_{i} \eta^{\rho}+\cdots+\frac{\partial g^{\sigma}}{\partial u_{i_{1} \cdots i_{m}}^{\rho}} D_{i_{1}} \cdots D_{i_{m}} \eta^{\rho}, \sigma=1, \ldots, N
\end{aligned}
$$

for $\eta^{\sigma}\left(t, \boldsymbol{x}, \boldsymbol{u}, \partial_{\boldsymbol{x}} \boldsymbol{u}, \ldots, \partial_{\boldsymbol{x}}^{p} \boldsymbol{u}\right)$, and

$$
\begin{aligned}
0=- & \mathcal{D}_{t} \omega_{\sigma}+\left(\mathcal{L}_{g}^{*}\right)_{\sigma}^{\rho} \omega_{\rho} \\
=- & \frac{\partial \omega_{\sigma}}{\partial t}+\left(\frac{\partial \omega_{\sigma}}{\partial u^{\rho}} g^{\rho}+\frac{\partial \omega_{\sigma}}{\partial u_{i}^{\rho}} D_{i} g^{\rho}+\cdots+\frac{\partial \omega_{\sigma}}{\partial u_{i_{1} \cdots i_{p}}^{\rho}} D_{i_{1}} \cdots D_{i_{p}} g^{\rho}\right) \\
& \quad+\frac{\partial g^{\rho}}{\partial u^{\sigma}} \omega_{\rho}-D_{i}\left(\frac{\partial g^{\rho}}{\partial u_{i}^{\sigma}} \omega_{\rho}\right)+\cdots+(-1)^{m} D_{i_{1}} \cdots D_{i_{m}}\left(\frac{\partial g^{\rho}}{\partial u_{i_{1} \cdots i_{m}}^{\sigma}} \omega_{\rho}\right), \sigma=1, \ldots, N
\end{aligned}
$$

for $\omega_{\sigma}\left(t, \boldsymbol{x}, \boldsymbol{u}, \partial_{x} \boldsymbol{u}, \ldots, \partial_{x}^{p} \boldsymbol{u}\right)$. The solutions of Eqs. 2.4) and 2.5) yield all symmetries and adjoint symmetries up to any given order $p$.

We now consider conservation laws.

Definition 2.1 A local conservation law of PDE system (1.1) is a divergence expression

$$
D_{t} \Phi^{t}\left(t, \boldsymbol{x}, \boldsymbol{u}, \partial \boldsymbol{u}, \ldots, \partial^{k} \boldsymbol{u}\right)+D_{i} \Phi^{i}\left(t, \boldsymbol{x}, \boldsymbol{u}, \partial \boldsymbol{u}, \ldots, \partial^{k} \boldsymbol{u}\right)=0
$$

for all solutions $\boldsymbol{u}(t, \boldsymbol{x})$ of Eq. (1.1); $\Phi^{t}$ and $\Phi^{i}$ are called the conserved densities.

The conservation equation (2.6) holds as an identity if, for all solutions $\boldsymbol{u}(t, \boldsymbol{x})$ of Eq. (1.1),

$$
\Phi^{t}=D_{i} \theta^{i}, \Phi^{i}=-D_{t} \theta^{i}+D_{j} \psi^{i j}
$$

for some expressions $\theta^{i}\left(t, \boldsymbol{x}, \boldsymbol{u}, \partial \boldsymbol{u}, \ldots, \partial^{k-1} \boldsymbol{u}\right), \psi^{i j}\left(t, \boldsymbol{x}, \boldsymbol{u}, \partial \boldsymbol{u}, \ldots, \partial^{k-1} \boldsymbol{u}\right)$ with $\psi^{i j}=$ $-\psi^{j i}$. Such conservation laws are trivial. Only the nontrivial conservation laws of the PDE system (1.1) are of interest.

Definition 2.2 A local conservation law (2.6) is nontrivial iff the conserved densities do not satisfy Eq. (2.7).

Any nontrivial conserved densities that agree to within trivial conserved densities are regarded as defining the same nontrivial conservation law. There is further freedom in 
the form of conserved densities since we are clearly free to replace $u_{t}^{\sigma}=-g^{\sigma}$ in $\Phi^{t}$ and $\Phi^{i}$ on the solution space of PDE system (1.1). Thus, without loss of generality we can consider $\Phi^{t}$ and $\Phi^{i}$ to depend only on $t, \boldsymbol{x}, \boldsymbol{u}$, and $\boldsymbol{x}$ derivatives of $\boldsymbol{u}$. We refer to this as the normal form of the conservation law,

$$
D_{t} \Phi^{t}\left(t, \boldsymbol{x}, \boldsymbol{u}, \partial_{\boldsymbol{x}} \boldsymbol{u}, \ldots, \partial_{\boldsymbol{x}}^{k} \boldsymbol{u}\right)+D_{i} \Phi^{i}\left(t, \boldsymbol{x}, \boldsymbol{u}, \partial_{\boldsymbol{x}} \boldsymbol{u}, \ldots, \partial_{\boldsymbol{x}}^{k} \boldsymbol{u}\right)=0
$$

for all solutions $\boldsymbol{u}(t, \boldsymbol{x})$ of PDE system (1.1). In normal form, the freedom corresponding to trivial conserved densities is given by

$$
\Phi^{t} \rightarrow \Phi^{t}+D_{i} \theta^{i}, \Phi^{i} \rightarrow \Phi^{i}-\mathcal{D}_{t} \theta^{i}+D_{j} \psi^{i j}
$$

where $\theta^{i}, \psi^{i j}=-\psi^{j i}$ do not depend on $\boldsymbol{u}_{t}$ and differential consequences.

All nontrivial local conservation laws (in normal form) can be shown to arise from multipliers on the PDEs (1.1) as follows. We move off the solution space of Eq. (1.1) and let $\boldsymbol{u}(t, \boldsymbol{x})$ be an arbitrary function of $t, \boldsymbol{x}$.

Definition 2.3 Multipliers for PDE system (1.1) are a set of expressions

$$
\left\{\Lambda_{1}\left(t, \boldsymbol{x}, \boldsymbol{u}, \partial \boldsymbol{u}, \ldots, \partial^{q} \boldsymbol{u}\right), \ldots, \Lambda_{N}\left(t, \boldsymbol{x}, \boldsymbol{u}, \partial \boldsymbol{u}, \ldots, \partial^{q} \boldsymbol{u}\right)\right\}
$$

satisfying

$$
\left(u_{t}^{\sigma}+g^{\sigma}\right) \Lambda_{\sigma}=D_{t} \tilde{\Phi}^{t}+D_{i} \tilde{\Phi}^{i}
$$

for some expressions $\tilde{\Phi}^{t}\left(t, \boldsymbol{x}, \boldsymbol{u}, \partial \boldsymbol{u}, \ldots, \partial^{k} \boldsymbol{u}\right)$ and $\tilde{\Phi}^{i}\left(t, \boldsymbol{x}, \boldsymbol{u}, \partial \boldsymbol{u}, \ldots, \partial^{k} \boldsymbol{u}\right)$ for all functions $\boldsymbol{u}(t, \boldsymbol{x})$.

Given a conservation law (2.8), consider $D_{t} \Phi^{t}+D_{i} \Phi^{i}$. Clearly this expression must be proportional to $u_{t}^{\sigma}+g^{\sigma}$ and its differential consequences in order to satisfy Eq. (2.8). The $u_{t}^{\sigma}$ terms arise only from

$$
D_{t} \Phi^{t}=\frac{\partial \Phi^{t}}{\partial t}+\frac{\partial \Phi^{t}}{\partial u^{\sigma}} u_{t}^{\sigma}+\frac{\partial \Phi^{t}}{\partial u_{i}^{\sigma}} u_{t i}^{\sigma}+\cdots+\frac{\partial \Phi^{t}}{\partial u_{i_{1} \cdots i_{k}}^{\sigma}} u_{t i_{1} \cdots i_{k}}^{\sigma}=\partial_{t} \Phi^{t}+\left(\mathcal{L}_{\Phi^{t}}\right)_{\sigma} u_{t}^{\sigma}
$$

where $\left(\mathcal{L}_{\Phi^{t}}\right)_{\sigma}=\left(\partial \Phi^{t} / \partial u^{\sigma}\right)+\left(\partial \Phi^{t} / \partial u_{i}^{\sigma}\right) D_{i}+\cdots+\left(\partial \Phi^{t} / \partial u_{i_{1} \cdots i_{k}}^{\sigma}\right) D_{i_{1}} \cdots D_{i_{k}}$ denotes the linearization operator of $\Phi^{t}$. To organize these terms we use the identities

$$
\begin{aligned}
\left(\mathcal{L}_{\Phi^{t}}\right)_{\sigma} u_{t}^{\sigma} & =\left(\mathcal{L}_{\Phi^{t}}\right)_{\sigma}\left(u_{t}^{\sigma}+g^{\sigma}\right)-\left(\mathcal{L}_{\Phi^{t}}\right)_{\sigma} g^{\sigma} \\
& =\left(u_{t}^{\sigma}+g^{\sigma}\right) \hat{E}_{u^{\sigma}}\left(\Phi^{t}\right)-\left(\mathcal{L}_{\Phi^{t}}\right)_{\sigma} g^{\sigma}+D_{i} \Gamma^{i}
\end{aligned}
$$

where $\Gamma^{i}$ is given by an expression proportional to $u_{t}^{\sigma}+g^{\sigma}$ (and differential consequences), and where

$$
\hat{E}_{u^{\sigma}}=\partial_{u^{\sigma}}-D_{i} \partial_{u_{i}^{\sigma}}+D_{i} D_{j} \partial_{u_{i j}^{\sigma}}+\cdots
$$

is a restricted Euler operator. Thus, we have

$$
D_{t} \Phi^{t}=\partial_{t} \Phi^{t}-\left(\mathcal{L}_{\Phi^{t}}\right)_{\sigma} g^{\sigma}+D_{i} \Gamma^{i}+\left(u_{t}^{\sigma}+g^{\sigma}\right) \hat{E}_{u^{\sigma}}\left(\Phi^{t}\right) .
$$

In order for the conservation equation (2.8) to hold, the terms $\partial_{t} \Phi^{t}-\left(\mathcal{L}_{\Phi^{t}}\right)_{\sigma} g^{\sigma}$ which do 
not involve $u_{t}^{\sigma}+g^{\sigma}$ must cancel $D_{i} \Phi^{i}$, and therefore we have

$$
D_{i} \Phi^{i}=-\partial_{t} \Phi^{t}+\left(\mathcal{L}_{\Phi^{t}}\right)_{\sigma} g^{\sigma}
$$

Then combining expressions (2.14) and (2.15) we obtain

$$
D_{t} \Phi^{t}+D_{i}\left(\Phi^{i}-\Gamma^{i}\right)=\left(u_{t}^{\sigma}+g^{\sigma}\right) \Lambda_{\sigma}
$$

with

$$
\Lambda_{\sigma}=\hat{E}_{u^{\sigma}}\left(\Phi^{t}\right), \sigma=1, \ldots, N .
$$

When $\boldsymbol{u}(t, \boldsymbol{x})$ is restricted to the solution space of PDE system (1.1), then $\Gamma^{i}$ vanishes and the divergence expression (2.16) reduces to the conservation equation (2.8).

Hence, the expressions $\left\{\hat{E}_{u^{\sigma}}\left(\Phi^{t}\right)\right\}$ define multipliers $\left\{\Lambda_{\sigma}\right\}$ yielding a conservation law (2.8). Furthermore, since $\Phi^{t}$ does not depend on $\boldsymbol{u}_{t}$ and its differential consequences, we see that each multiplier expression $\Lambda_{\sigma}$ is a function only of $t, \boldsymbol{x}, \boldsymbol{u}$, and $\boldsymbol{x}$ derivatives of $\boldsymbol{u}$. Most important, these expressions $\Lambda_{\sigma}$ are invariant under a change in $\Phi^{t}$ by a trivial conserved density (2.9) since $\hat{E}_{u^{\sigma}}$ annihilates divergences $D_{i} \theta^{i}$ where $\theta^{i}$ depends on $t, \boldsymbol{x}, \boldsymbol{u}$ and $\boldsymbol{x}$ derivatives of $\boldsymbol{u}$. (In particular, if $\Phi^{t}$ in normal form is trivial, then $\Lambda_{\sigma}$ is identically zero, and conversely.) Thus we have the following result.

Theorem 2.4 For the Cauchy-Kovalevskaya PDE system (1.1), every nontrivial conservation law in normal form (2.8) is uniquely characterized by a set of multipliers $\left\{\Lambda_{\sigma}\right\}$ with no dependence on $\boldsymbol{u}_{t}$ and differential consequences, satisfying the relations (2.10) and (2.17) holding for all functions $\boldsymbol{u}(t, \boldsymbol{x})$.

From this result it is natural to define the order of a conservation law (2.8) as the order of the highest $\boldsymbol{x}$ derivative of $\boldsymbol{u}$ in its multipliers (2.17).

Theorem 2.4 is the starting point for an effective approach to find conservation laws of PDE system (1.1) by use of multipliers. The standard determining condition [11 for multiplier expressions $\Lambda_{\sigma}\left(t, \boldsymbol{x}, \boldsymbol{u}, \partial_{\boldsymbol{x}} \boldsymbol{u}, \ldots, \partial_{\boldsymbol{x}}^{p} \boldsymbol{u}\right)$ arises from the definition (2.10) by the well-known result that divergence expressions are characterized by annihilation under the full Euler operator

$$
E_{u^{\sigma}}=\partial_{u^{\sigma}}-D_{i} \partial_{u_{i}^{\sigma}}-D_{t} \partial_{u_{t}^{\sigma}}+D_{i} D_{j} \partial_{u_{i j}^{\sigma}}+D_{t} D_{j} \partial_{u_{t j}^{\sigma}}+\cdots
$$

This yields (by a straightforward calculation)

$$
0=E_{u^{\sigma}}\left(u_{t}^{\rho} \Lambda_{\rho}+g^{\rho} \Lambda_{\rho}\right)=-D_{t} \Lambda_{\sigma}+\left(\mathcal{L}_{g}^{*}\right)_{\sigma}^{\rho} \Lambda_{\rho}+\left(\mathcal{L}_{\Lambda}^{*}\right)_{\sigma \rho}\left(u_{t}^{\rho}+g^{\rho}\right), \sigma=1, \ldots, N
$$

where $\left(\mathcal{L}_{\Lambda}^{*}\right)_{\sigma \rho}$ is the adjoint operator of the linearization operator $\left(\mathcal{L}_{\Lambda}\right)_{\sigma \rho}$ defined by

$$
\left(\mathcal{L}_{\Lambda}\right)_{\sigma \rho} V^{\rho}=\frac{\partial \Lambda_{\sigma}}{\partial u^{\rho}} V^{\rho}+\frac{\partial \Lambda_{\sigma}}{\partial u_{i}^{\rho}} D_{i} V^{\rho}+\cdots+\frac{\partial \Lambda_{\sigma}}{\partial u_{i_{1} \cdots i_{p}}^{\rho}} D_{i_{1}} \cdots D_{i_{p}} V^{\rho}
$$

and

$$
\left(\mathcal{L}_{\Lambda}^{*}\right)_{\rho \sigma} W^{\sigma}=\frac{\partial \Lambda_{\sigma}}{\partial u^{\rho}} W^{\sigma}-D_{i}\left(\frac{\partial \Lambda_{\sigma}}{\partial u_{i}^{\rho}} W^{\sigma}\right)+\cdots+(-1)^{p} D_{i_{1}} \cdots D_{i_{p}}\left(\frac{\partial \Lambda_{\sigma}}{\partial u_{i_{1} \cdots i_{p}}^{\rho}} W^{\sigma}\right)
$$


acting on arbitrary functions $V^{\rho}, W^{\sigma}$. Here the determining condition (2.19) is required to hold for all functions $\boldsymbol{u}(t, \boldsymbol{x})$, i.e., this is necessary and sufficient for $u_{t}^{\rho} \Lambda_{\rho}+g^{\rho} \Lambda_{\rho}$ to be a divergence expression. We give a simple direct proof in Sec. 2.3.

We now show how to convert the determining condition for $\Lambda_{\sigma}$ into a system of determining equations that allow one to work entirely on the space of solutions of PDE system (1.1) to find $\Lambda_{\sigma}$. Furthermore, we show that the resulting determining system consists of the adjoint symmetry determining equation (2.5) augmented by extra determining equations giving necessary and sufficient conditions for an adjoint symmetry to be a set of multipliers yielding a conservation law.

\subsection{Conservation law determining system}

In the determining condition (2.19) for $\Lambda_{\sigma}\left(t, \boldsymbol{x}, \boldsymbol{u}, \partial_{\boldsymbol{x}} \boldsymbol{u}, \ldots, \partial_{\boldsymbol{x}}^{p} \boldsymbol{u}\right)$ of order $p$ consider the terms involving $u_{t}^{\sigma}$. These terms arise just from $D_{t} \Lambda_{\sigma}$ and $\left(\mathcal{L}_{\Lambda}^{*}\right)_{\sigma \rho} u_{t}^{\rho}$, and so it follows that Eq. (2.19) is a linear polynomial in $u_{t}^{\sigma}$ and differential consequences of $u_{t}^{\sigma}$ with respect to $\boldsymbol{x}$. Since $u^{\sigma}$ is required to be an arbitrary function of $t$ and $\boldsymbol{x}$, Eq. (2.19) splits into separate equations given by the coefficients of $u_{t}^{\sigma}, u_{t i}^{\sigma}$, etc. It is convenient to organize this splitting in terms of $u_{t}^{\sigma}+g^{\sigma}=G^{\sigma}$ and differential consequences $u_{t i}^{\sigma}+D_{i} g^{\sigma}=D_{i} G^{\sigma}$, etc., which we refer to as the leading terms (all other terms in the splitting are then referred to as non-leading). Then the leading and non-leading terms in the splitting must vanish separately.

To carry out the splitting of $D_{t} \Lambda_{\sigma}$, we use the identity

$$
D_{t}=\mathcal{D}_{t}+\left(u_{t}^{\rho}+g^{\rho}\right) \partial_{u^{\rho}}+\left(u_{t i}^{\rho}+D_{i} g^{\rho}\right) \partial_{u_{i}^{\rho}}+\cdots
$$

which yields $D_{t} \Lambda_{\sigma}=\mathcal{D}_{t} \Lambda_{\sigma}+\left(\mathcal{L}_{\Lambda}\right)_{\sigma \rho} G^{\rho}$.

Consequently, the non-leading terms in Eq. (2.19) are given by

$$
\begin{aligned}
0= & -\mathcal{D}_{t} \Lambda_{\sigma}+\left(\mathcal{L}_{g}^{*}\right)_{\sigma}^{\rho} \Lambda_{\rho} \\
=-\frac{\partial \Lambda_{\sigma}}{\partial t}+ & \left(\frac{\partial \Lambda_{\sigma}}{\partial u^{\rho}} g^{\rho}+\frac{\partial \Lambda_{\sigma}}{\partial u_{i}^{\rho}} D_{i} g^{\rho}+\cdots+\frac{\partial \Lambda_{\sigma}}{\partial u_{i_{1} \cdots i_{p}}^{\rho}} D_{i_{1}} \cdots D_{i_{p}} g^{\rho}\right) \\
& \quad+\frac{\partial g^{\rho}}{\partial u^{\sigma}} \Lambda_{\rho}-D_{i}\left(\frac{\partial g^{\rho}}{\partial u_{i}^{\sigma}} \Lambda_{\rho}\right)+\cdots+(-1)^{m} D_{i_{1}} \cdots D_{i_{m}}\left(\frac{\partial g^{\rho}}{\partial u_{i_{1} \cdots i_{m}}^{\sigma}} \Lambda_{\rho}\right), \\
& \sigma=1, \ldots, N .
\end{aligned}
$$

This is the adjoint symmetry equation (2.5) with $\omega_{\sigma}=\Lambda_{\sigma}$.

The leading terms in Eq. (2.19) are given by

$$
0=-\left(\mathcal{L}_{\Lambda}\right)_{\sigma \rho} G^{\rho}+\left(\mathcal{L}_{\Lambda}^{*}\right)_{\sigma \rho} G^{\rho}, \sigma=1, \ldots, N .
$$

which we call the adjoint invariance condition on $\Lambda_{\sigma}$. Now since $u^{\sigma}$ is required to be an arbitrary function of $t$ and $\boldsymbol{x}$, we observe that Eq. (2.23) splits into separate equations 
given by the coefficients of $G^{\sigma}, D_{i} G^{\sigma}, \ldots, D_{i_{1}} \cdots D_{i_{p}} G^{\sigma}$ :

$$
\begin{aligned}
& \begin{array}{l}
0=(-1)^{p+1} \\
\frac{\partial \Lambda_{\sigma}}{\partial u_{i_{1} \cdots i_{p}}^{\rho}}+\frac{\partial \Lambda_{\rho}}{\partial u_{i_{1} \cdots i_{p}}^{\sigma}}
\end{array} \\
& 0=(-1)^{q+1} \frac{\partial \Lambda_{\sigma}}{\partial u_{i_{1} \cdots i_{q}}^{\rho}}+\frac{\partial \Lambda_{\rho}}{\partial u_{i_{1} \cdots i_{q}}^{\sigma}}-C_{q}^{q+1} D_{i_{q+1}} \frac{\partial \Lambda_{\rho}}{\partial u_{i_{1} \cdots i_{q+1}}^{\sigma}}+\cdots \\
& \quad+(-1)^{p-q} C_{q}^{p} D_{i_{q+1}} \cdots D_{i_{p}} \frac{\partial \Lambda_{\rho}}{\partial u_{i_{1} \cdots i_{p}}^{\sigma}}, \quad q=1, \ldots, p-1 \\
& 0=-\frac{\partial \Lambda_{\sigma}}{\partial u^{\rho}}+\frac{\partial \Lambda_{\rho}}{\partial u^{\sigma}}-D_{i} \frac{\partial \Lambda_{\rho}}{\partial u_{i}^{\sigma}}+\cdots+(-1)^{p} D_{i_{1}} \cdots D_{i_{p}} \frac{\partial \Lambda_{\rho}}{\partial u_{i_{1} \cdots i_{p}}^{\sigma}} \\
& \sigma=1, \ldots, N ; \rho=1, \ldots, N
\end{aligned}
$$

where $C_{q}^{r}=\frac{r !}{q !(r-q) !}$. This establishes the following important splitting result.

Lemma 2.5 For $\Lambda_{\sigma}$ with no dependence on $\boldsymbol{u}_{t}$ and differential consequences, the Euler operator equation (2.19) is equivalent to the split system of equations (2.22) and (2.24), which are required to hold for all functions $\boldsymbol{u}(t, \boldsymbol{x})$.

Consequently, by combining Lemma 2.5 and Theorem 2.4, we see that Eqs. (2.22) and (2.24) constitute a necessary and sufficient determining system for finding multipliers $\left\{\Lambda_{\sigma}\right\}$. The number of equations in this system is $\frac{N^{2}(n+p-1) !}{n !(p-1) !}+\frac{N\left(N-(-1)^{p}\right)}{2} \frac{(n+p) !}{n ! p !}$.

Theorem 2.6 For the Cauchy-Kovalevskaya PDE system (1.1), the multipliers for all nontrivial conservation laws in normal form (2.8) up to any given order $p$ are the solutions $\Lambda_{\sigma}\left(t, \boldsymbol{x}, \boldsymbol{u}, \partial_{\boldsymbol{x}} \boldsymbol{u}, \ldots, \partial_{\boldsymbol{x}}^{p} \boldsymbol{u}\right)$ of the determining system consisting of the adjoint symmetry determining equation (2.29) augmented by the extra determining equations (2.24). In particular, Eq. (2.24) gives necessary and sufficient conditions for an adjoint symmetry to be a set of multipliers.

In deriving the determining system for $\Lambda_{\sigma}$, we have eliminated $\boldsymbol{u}_{t}$ and its differential consequences. As a result, one is able to work equivalently on the space of solutions of the PDE system (1.1) in order to solve the determining system to find $\Lambda_{\sigma}$. In particular, the same algorithmic procedures which one uses to solve determining equations for symmetries can be used to solve the determining system for multipliers. Moreover, there is freedom in mixing the order of solving the determining equations in this system. A direct (naive) approach is to solve the adjoint symmetry determining equation first, then check which of these adjoint symmetries satisfy the extra determining equations. As illustrated in the examples in Part I, a more effective approach is to use the extra determining equations first.

\section{Remarks on the extra determining equations:}

There is a simple interpretation of the extra determining equations (2.24). From relation (2.17) between multipliers and conserved densities, we observe that $\Lambda_{\sigma}$ is a variational expression (i.e. it arises as an Euler-Lagrange expression from $\Phi^{t}$ ). The well-known 
necessary and sufficient (Helmholtz) conditions [1] for an expression to be variational are that its linearization operator is self-adjoint, and thus $\Lambda_{\sigma}$ is a variational expression if and only if it satisfies [7, 8]

$$
\left(\mathcal{L}_{\Lambda}\right)_{\sigma \rho}=\left(\mathcal{L}_{\Lambda}^{*}\right)_{\sigma \rho}, \sigma, \rho=1, \ldots, N
$$

The operator equation (2.25) is a linear polynomial in $D_{i}$ of degree $p$. We easily find that if it is decomposed into separate equations given by the coefficients of the polynomial, then the resulting equations are the same as the determining equations (2.24).

Corollary 2.7 Multipliers for any first-order Cauchy-Kovalevskaya PDE system are completely characterized as adjoint symmetries with a variational form.

Moreover, it is interesting to note that the determining equations 2.24) take the same form regardless of $g^{\sigma}$ for all first-order Cauchy-Kovalevskaya PDE systems (1.1).

\subsection{Conservation law construction formula}

We now give an integral formula that constructs the conserved densities $\Phi^{t}$ and $\Phi^{i}$ for any nontrivial conservation law in normal form (2.8) in terms of its multipliers $\left\{\Lambda_{\sigma}\right\}$. The formula makes use of the identities [1]

$$
\begin{aligned}
& W_{\sigma}\left(\mathcal{L}_{g}\right)_{\rho}^{\sigma} V^{\rho}-V^{\rho}\left(\mathcal{L}_{g}^{*}\right)_{\rho}^{\sigma} W_{\sigma}=D_{i} S^{i}[V, W ; g], \\
& W^{\sigma}\left(\mathcal{L}_{\Lambda}\right)_{\sigma \rho} V^{\rho}-V^{\rho}\left(\mathcal{L}_{\Lambda}^{*}\right)_{\sigma \rho} W^{\sigma}=D_{i} S^{i}[V, W ; \Lambda],
\end{aligned}
$$

where

$$
\begin{aligned}
& S^{i}[V, W ; g]=\sum_{\ell=0}^{m-1} \sum_{k=0}^{m-\ell-1}(-1)^{k}\left(D_{i_{1}} \cdots D_{i_{\ell}} V^{\rho}\right) D_{j_{1}} \cdots D_{j_{k}}\left(W_{\sigma} \frac{\partial g^{\sigma}}{\partial u_{i i_{1} \cdots i_{k} j_{1} \cdots j_{\ell}}^{\rho}}\right), \\
& S^{i}[V, W ; \Lambda]=\sum_{\ell=0}^{p-1} \sum_{k=0}^{p-\ell-1}(-1)^{k}\left(D_{i_{1}} \cdots D_{i_{\ell}} V^{\rho}\right) D_{j_{1}} \cdots D_{j_{k}}\left(W^{\sigma} \frac{\partial \Lambda_{\sigma}}{\partial u_{i i_{1} \cdots i_{k} j_{1} \cdots j_{\ell}}^{\rho}}\right),
\end{aligned}
$$

which are trilinear expressions derived by manipulation of the linearization operators and adjoint operators. (Note, the terms in Eqs. (2.28) and (2.29) with $\ell=0$ or $k=0$ are understood to involve no derivatives of $V$ and $W$, respectively.)

To set up the formula, we first let

$$
u_{(\lambda)}^{\sigma}=\lambda u^{\sigma}+(1-\lambda) \tilde{u}^{\sigma}
$$

where $\left\{\tilde{u}^{\sigma}\right\}$ are any functions of $t, \boldsymbol{x}$. This defines a one-parameter $\lambda$ family of functions with $u_{(1)}^{\sigma}=u^{\sigma}$ and $u_{(0)}^{\sigma}=\tilde{u}^{\sigma}$. Then we let

$$
\begin{aligned}
& \Lambda_{\rho}\left[\boldsymbol{u}_{(\lambda)}\right]=\Lambda_{\rho}\left(t, \boldsymbol{x}, \boldsymbol{u}_{(\lambda)}, \partial_{\boldsymbol{x}} \boldsymbol{u}_{(\lambda)}, \ldots, \partial_{\boldsymbol{x}}^{p} \boldsymbol{u}_{(\lambda)}\right) \\
& g^{\rho}\left[\boldsymbol{u}_{(\lambda)}\right]=g^{\rho}\left(t, \boldsymbol{x}, \boldsymbol{u}_{(\lambda)}, \partial_{\boldsymbol{x}} \boldsymbol{u}_{(\lambda)}, \ldots, \partial_{\boldsymbol{x}}^{m} \boldsymbol{u}_{(\lambda)}\right)
\end{aligned}
$$




$$
K(t, \boldsymbol{x})=\left.\left(\left(u_{(\lambda) t}^{\rho}+g^{\rho}\left[\boldsymbol{u}_{(\lambda)}\right]\right) \Lambda_{\rho}\left[\boldsymbol{u}_{(\lambda)}\right]\right)\right|_{\lambda=0} .
$$

Theorem 2.8 For the Cauchy-Kovalevskaya PDE system (1.), the conserved densities of any nontrivial conservation law in normal form are given in terms of the multipliers by

$$
\begin{aligned}
& \Phi^{t}=\int_{0}^{1} d \lambda\left(u^{\sigma}-\tilde{u}^{\sigma}\right) \Lambda_{\sigma}\left[\boldsymbol{u}_{(\lambda)}\right]+t \int_{0}^{1} d \lambda K(\lambda t, \lambda \boldsymbol{x}) \\
& \Phi^{i}=x^{i} \int_{0}^{1} d \lambda \lambda^{n} K(\lambda t, \lambda \boldsymbol{x})+\int_{0}^{1} d \lambda\left(S^{i}\left[\boldsymbol{u}-\tilde{\boldsymbol{u}}, \boldsymbol{\Lambda}\left[\boldsymbol{u}_{(\lambda)}\right] ; \boldsymbol{g}\left[\boldsymbol{u}_{(\lambda)}\right]\right]\right. \\
& \left.\quad+S^{i}\left[\boldsymbol{u}-\tilde{\boldsymbol{u}}, \boldsymbol{g}\left[\boldsymbol{u}_{(\lambda)}\right]-\lambda \boldsymbol{g}[\boldsymbol{u}]+(1-\lambda) \tilde{\boldsymbol{u}}_{t} ; \boldsymbol{\Lambda}\left[\boldsymbol{u}_{(\lambda)}\right]\right]\right)
\end{aligned}
$$

In applying the construction formula (2.34) and (2.35), we must fix a choice for the functions $\left\{\tilde{u}^{\sigma}\right\}$. If the expressions $\Lambda_{\sigma}$ and $g^{\sigma}$ are nonsingular for $u^{\sigma}=0$, then we can choose $\tilde{u}^{\sigma}=0$ and this simplifies the integrals. Moreover, if $\tilde{u}^{\sigma}=u^{\sigma}=0$ satisfies the PDE system (1.1), then the $K$ integrals vanish.

In the case when the expressions $\Lambda_{\sigma}$ and $g^{\sigma}$ are singular at $u^{\rho}=0$ (for some $\rho=$ $1, \ldots, N)$, we must choose $\tilde{u}^{\rho} \neq 0$ such that the expressions $\Lambda_{\sigma}[\tilde{\boldsymbol{u}}]$ and $g^{\sigma}[\tilde{\boldsymbol{u}}]$ are nonsingular. It is sufficient to fix a simple choice of $\tilde{u}^{\rho}$ such that the integrals converge. Any change in the choice of $\tilde{u}^{\rho}$ changes the conserved densities only by a trivial conserved density (2.9).

A simple proof of Theorem 2.8 is given in Sec. 2.3.

\subsection{Proofs of Main Equations}

Recall that, for first-order Cauchy-Kovalevskaya PDE systems (1.1), the proof of the determining system (2.24) for conservation law multipliers in Theorem 2.6 reduces, by Lemma 2.5, to the determining condition (2.19) involving the Euler operator. To conclude this section, we present a simple, direct proof of this determining condition (2.19) together with the construction formula (2.34) and (2.35) for corresponding conserved densities in Theorem 2.8. The proof of Eq. (2.19) is based on an identity for linearization of the multiplier equation (2.10).

We let

$$
u_{(\lambda)}^{\sigma}=(\lambda-1) v^{\sigma}+u^{\sigma}
$$

be a one-parameter family of functions with $u_{(1)}^{\sigma}=u^{\sigma}$ being an arbitrary function, and with $\partial u_{(\lambda)}^{\sigma} / \partial \lambda=v^{\sigma}$ for any functions $v^{\sigma}(t, \boldsymbol{x})$.

Proposition 2.9 For any given expressions $\Lambda_{\sigma}[\boldsymbol{u}]=\Lambda_{\sigma}\left(t, \boldsymbol{x}, \boldsymbol{u}, \partial_{\boldsymbol{x}} \boldsymbol{u}, \ldots, \partial_{\boldsymbol{x}}^{p} \boldsymbol{u}\right), \tilde{\Phi}^{t}[\boldsymbol{u}]=$ $\tilde{\Phi}^{t}\left(t, \boldsymbol{x}, \boldsymbol{u}, \partial_{\boldsymbol{x}} \boldsymbol{u}, \ldots, \partial_{\boldsymbol{x}}^{k} \boldsymbol{u}\right)$ and $\tilde{\Phi}^{i}[\boldsymbol{u}]=\tilde{\Phi}^{i}\left(t, \boldsymbol{x}, \boldsymbol{u}, \partial_{\boldsymbol{x}} \boldsymbol{u}, \ldots, \partial_{\boldsymbol{x}}^{k} \boldsymbol{u}\right)$, the following identities hold by direct calculation:

$$
\text { (i) } \begin{aligned}
& \frac{\partial}{\partial \lambda}\left(\left(u_{(\lambda) t}^{\sigma}+g^{\sigma}\left[\boldsymbol{u}_{(\lambda)}\right]\right) \Lambda_{\sigma}\left[\boldsymbol{u}_{(\lambda)}\right]\right) \\
& =\left(v_{t}^{\sigma}+\left(\mathcal{L}_{\boldsymbol{g}\left[\boldsymbol{u}_{(\lambda)}\right]}\right)_{\rho}^{\sigma} v^{\rho}\right) \Lambda_{\sigma}\left[\boldsymbol{u}_{(\lambda)}\right]+\left(u_{(\lambda) t}^{\sigma}+g^{\sigma}\left[\boldsymbol{u}_{(\lambda)}\right]\right)\left(\mathcal{L}_{\boldsymbol{\Lambda}\left[\boldsymbol{u}_{(\lambda)}\right]}\right)_{\sigma \rho} v^{\rho}
\end{aligned}
$$




$$
\begin{aligned}
& =v^{\sigma}\left(-D_{t} \Lambda_{\sigma}\left[\boldsymbol{u}_{(\lambda)}\right]+\left(\mathcal{L}_{\boldsymbol{g}\left[\boldsymbol{u}_{(\lambda)}\right]}^{*}\right)_{\sigma}^{\rho} \Lambda_{\rho}\left[\boldsymbol{u}_{(\lambda)}\right]+\left(\mathcal{L}_{\boldsymbol{\Lambda}\left[\boldsymbol{u}_{(\lambda)}\right]}^{*}\right)_{\sigma \rho}\left(u_{(\lambda) t}^{\rho}+g^{\rho}\left[\boldsymbol{u}_{(\lambda)}\right]\right)\right) \\
& +D_{t}\left(v^{\sigma} \Lambda_{\sigma}\left[\boldsymbol{u}_{(\lambda)}\right]\right)+D_{i}\left(S^{i}\left[\boldsymbol{v}, \boldsymbol{\Lambda}\left[\boldsymbol{u}_{(\lambda)}\right] ; \boldsymbol{g}\left[\boldsymbol{u}_{(\lambda)}\right]\right]\right. \\
& \left.\quad+S^{i}\left[\boldsymbol{v}, \boldsymbol{u}_{(\lambda) t}+\boldsymbol{g}\left[\boldsymbol{u}_{(\lambda)}\right] ; \boldsymbol{\Lambda}\left[\boldsymbol{u}_{(\lambda)}\right]\right]\right)
\end{aligned}
$$

where $S^{i}$ denotes the trilinear expressions given by Eqs. (2.28) and (2.29);

$$
\text { (ii) } \frac{\partial}{\partial \lambda}\left(D_{t} \tilde{\Phi}^{t}\left[\boldsymbol{u}_{(\lambda)}\right]+D_{i} \tilde{\Phi}^{i}\left[\boldsymbol{u}_{(\lambda)}\right]\right)=D_{t}\left(\left(\mathcal{L}_{\tilde{\Phi}^{t}\left[\boldsymbol{u}_{(\lambda)}\right]}\right)_{\sigma} v^{\sigma}\right)+D_{i}\left(\left(\mathcal{L}_{\tilde{\Phi}\left[\boldsymbol{u}_{(\lambda)}\right]}\right)_{\sigma}^{i} v^{\sigma}\right)
$$

where $\left(\mathcal{L}_{\tilde{\Phi}^{t}}\right)_{\sigma}$ and $\left(\mathcal{L}_{\tilde{\Phi}}\right)_{\sigma}^{i}$ denote the linearization operators of $\tilde{\Phi}^{t}$ and $\tilde{\Phi}^{i}$ respectively.

\section{Proof of the multiplier determining condition and conserved density con- struction formula:}

Suppose $\Phi^{t}, \Phi^{i}$ are conserved densities of a conservation law in normal form (2.8). From Theorem 2.4 the multipliers for the conservation law are given by $\Lambda_{\sigma}=\hat{E}_{u^{\sigma}}\left(\Phi^{t}\right)$ satisfying the multiplier equation (2.10) with $\tilde{\Phi}^{t}=\Phi^{t}, \tilde{\Phi}^{i}=\Phi^{i}-\Gamma^{i}$.

Since the multiplier equation (2.10) holds for all functions $u^{\sigma}(t, \boldsymbol{x})$, it must hold for the one-parameter family $u_{(\lambda)}^{\sigma}$. We now take the derivative of the resulting left-side and right-side expressions of Eq. 2.10) with respect to $\lambda$. By Proposition 2.9, on the leftside we obtain Eq. (2.37), while on the right-side we directly obtain Eq. (2.38). These expressions (2.37) and (2.38) are equal for all functions $v^{\sigma}(t, \boldsymbol{x})$ and therefore hold iff the terms multiplying $v^{\sigma}$ vanish and the total derivative terms involving $v^{\sigma}$ are separately equal (by considering the terms $v_{t}^{\sigma}, v_{i}^{\sigma}$ ). From the terms multiplying $v^{\sigma}$ we have

$$
0=-D_{t} \Lambda_{\sigma}\left[\boldsymbol{u}_{(\lambda)}\right]+\left(\mathcal{L}_{\boldsymbol{g}\left[\boldsymbol{u}_{(\lambda)}\right.}^{*}\right]_{\sigma}^{\rho} \Lambda_{\rho}\left[\boldsymbol{u}_{(\lambda)}\right]+\left(\mathcal{L}_{\boldsymbol{\Lambda}\left[\boldsymbol{u}_{(\lambda)}\right]}^{*}\right)_{\sigma \rho}\left(u_{(\lambda) t}^{\rho}+g^{\rho}\left[\boldsymbol{u}_{(\lambda)}\right]\right)
$$

This reduces when $\lambda=1$ to Eq. (2.19) and hence $\left\{\Lambda_{\sigma}\right\}$ is a solution of the determining condition (2.19).

Conversely, suppose $\left\{\Lambda_{\sigma}\right\}$ is a solution of the determining condition (2.19). Then, by combining the two identities in Proposition 2.9, we see $\Lambda_{\sigma}$ satisfies the linearized multiplier equation

$$
\frac{\partial}{\partial \lambda}\left(\left(u_{(\lambda) t}^{\sigma}+g^{\sigma}\left[\boldsymbol{u}_{(\lambda)}\right]\right) \Lambda_{\sigma}\left[\boldsymbol{u}_{(\lambda)}\right]\right)=D_{t} \frac{\partial}{\partial \lambda} \tilde{\Phi}^{t}\left[\boldsymbol{u}_{(\lambda)}\right]+D_{i} \frac{\partial}{\partial \lambda} \tilde{\Phi}^{i}\left[\boldsymbol{u}_{(\lambda)}\right]
$$

with $\partial \tilde{\Phi}^{t}\left[\boldsymbol{u}_{(\lambda)}\right] / \partial \lambda$ and $\partial \tilde{\Phi}^{i}\left[\boldsymbol{u}_{(\lambda)}\right] / \partial \lambda$ defined by

$$
\begin{aligned}
& \left(\mathcal{L}_{\tilde{\Phi}^{t}\left[\boldsymbol{u}_{(\lambda)}\right]}\right)_{\sigma} v^{\sigma}=v^{\sigma} \Lambda_{\sigma}\left[\boldsymbol{u}_{(\lambda)}\right]+D_{i} \theta^{i} \\
& \left(\mathcal{L}_{\tilde{\Phi}\left[\boldsymbol{u}_{(\lambda)}\right]}\right)_{\sigma}^{i} v^{\sigma}=\left(S^{i}\left[\boldsymbol{v}, \boldsymbol{\Lambda}\left[\boldsymbol{u}_{(\lambda)}\right] ; \boldsymbol{g}\left[\boldsymbol{u}_{(\lambda)}\right]\right]+S^{i}\left[\boldsymbol{v}, \boldsymbol{u}_{(\lambda) t}+\boldsymbol{g}\left[\boldsymbol{u}_{(\lambda)}\right] ; \boldsymbol{\Lambda}\left[\boldsymbol{u}_{(\lambda)}\right]\right]\right)-D_{t} \theta^{i}+D_{j} \psi^{i j}
\end{aligned}
$$

for some expressions $\theta^{i}, \psi^{i j}=-\psi^{j i}$. We now undo the linearization to obtain the multiplier equation (2.10) by integrating with respect to $\lambda$ as follows. We set $v^{\sigma}=u^{\sigma}-\tilde{u}^{\sigma}$, and so

$$
u_{(\lambda)}^{\sigma}=\lambda\left(u^{\sigma}-\tilde{u}^{\sigma}\right)+\tilde{u}^{\sigma} .
$$


Then we use the fundamental theorem of calculus to obtain

$$
\left(u_{t}^{\sigma}+g^{\sigma}[\boldsymbol{u}]\right) \Lambda_{\sigma}[\boldsymbol{u}]=D_{t} \tilde{\Phi}^{t}[\boldsymbol{u}]+D_{i} \tilde{\Phi}^{i}[\boldsymbol{u}]+\left(\tilde{u}_{t}^{\sigma}+g^{\sigma}[\tilde{\boldsymbol{u}}]\right) \Lambda_{\sigma}[\tilde{\boldsymbol{u}}]-D_{t} \tilde{\Phi}^{t}[\tilde{\boldsymbol{u}}]-D_{i} \tilde{\Phi}^{i}[\tilde{\boldsymbol{u}}]
$$

where

$$
\begin{aligned}
\tilde{\Phi}^{t}[\boldsymbol{u}]=\tilde{\Phi}^{t}[\tilde{\boldsymbol{u}}]+ & \int_{0}^{1} d \lambda\left(u^{\sigma}-\tilde{u}^{\sigma}\right) \Lambda_{\sigma}\left[\boldsymbol{u}_{(\lambda)}\right], \\
\tilde{\Phi}^{i}[\boldsymbol{u}]=\tilde{\Phi}^{i}[\tilde{\boldsymbol{u}}]+ & \int_{0}^{1} d \lambda\left(S^{i}\left[\boldsymbol{u}-\tilde{\boldsymbol{u}}, \boldsymbol{\Lambda}\left[\boldsymbol{u}_{(\lambda)}\right] ; \boldsymbol{g}\left[\boldsymbol{u}_{(\lambda)}\right]\right]\right. \\
& \left.+S^{i}\left[\boldsymbol{u}-\tilde{\boldsymbol{u}}, \boldsymbol{u}_{(\lambda) t}+\boldsymbol{g}\left[\boldsymbol{u}_{(\lambda)}\right] ; \boldsymbol{\Lambda}\left[\boldsymbol{u}_{(\lambda)}\right]\right]\right),
\end{aligned}
$$

to within trivial conserved densities. Since Eq. (2.44) holds for all $u(t, \boldsymbol{x})$, while $\tilde{u}(t, \boldsymbol{x})$ is fixed, we must have

$$
D_{t} \tilde{\Phi}^{t}[\tilde{\boldsymbol{u}}]+D_{i} \tilde{\Phi}^{i}[\tilde{\boldsymbol{u}}]=\left(\tilde{u}_{t}^{\sigma}+g^{\sigma}[\tilde{\boldsymbol{u}}]\right) \Lambda_{\sigma}[\tilde{\boldsymbol{u}}]=K(t, \boldsymbol{x}) .
$$

It is then simple to check that Eq. (2.47) is satisfied identically by

$$
\tilde{\Phi}^{t}[\tilde{\boldsymbol{u}}]=t \int_{0}^{1} d \lambda K(\lambda t, \lambda \boldsymbol{x}), \tilde{\Phi}^{i}[\tilde{\boldsymbol{u}}]=x^{i} \int_{0}^{1} d \lambda K(\lambda t, \lambda \boldsymbol{x}) .
$$

Thus, we find from Eq. 2.44) that $\left\{\Lambda_{\sigma}\right\}$ satisfies the multiplier equation (2.10), with conserved densities given by Eqs. (2.45) to (2.48). Hence, by Theorem 2.4, $\Lambda_{\sigma}$ are multipliers for a conservation law in normal form (2.8).

To obtain the construction formula (2.34) and (2.35) for the conserved densities, we move onto the solution space of Eq. (1.1) and substitute $u_{(\lambda) t}^{\sigma}=-\lambda g^{\sigma}[\boldsymbol{u}]+(1-\lambda) \tilde{u}_{t}^{\sigma}$ into Eqs. (2.45) and (2.46). The expressions $\tilde{\Phi}^{t}[\boldsymbol{u}]$ and $\tilde{\Phi}^{i}[\boldsymbol{u}]$ directly reduce to the formula for $\Phi^{t}$ and $\Phi^{i}$.

\section{Treatment of Nth order scalar PDEs}

Here we exhibit the conservation law determining system and construction formula for scalar PDEs of any order with one dependent variable $u$ and $n+1$ independent variables $t, \boldsymbol{x}=\left(x^{1}, \ldots, x^{n}\right)$. We work directly with the scalar PDE expressed in an $N$ th order Cauchy-Kovalevskaya form

$$
G=\frac{\partial^{N} u}{\partial t^{N}}+g\left(t, x, u, \partial u, \ldots, \partial^{m} u\right)=0
$$

where in this section $\partial^{q} u$ now denotes all derivatives of $u$ of order $q$, excluding $t$ derivatives of $u$ of order $q \geq N$ and their differential consequences (i.e. the PDE is written so that the $t$ derivatives of $u$ of highest order appear in solved form).

Clearly, without loss of generality, for conservation laws we are free to eliminate $N$ th order $t$ derivatives of $u$ (and differential consequences) in considering conserved densities.

Definition 3.1 A local conservation law in normal form for a Cauchy-Kovalevskaya scalar PDE (3.1) is a divergence expression

$$
D_{t} \Phi^{t}\left(t, \boldsymbol{x}, u, \partial u, \ldots, \partial^{k} u\right)+D_{i} \Phi^{i}\left(t, \boldsymbol{x}, u, \partial u, \ldots, \partial^{k} u\right)=0
$$


holding for all solutions $u(t, \boldsymbol{x})$ of Eq. (3.1).

A conservation law (3.2) is trivial if it holds as an identity (2.7) for some expressions $\theta^{i}\left(t, \boldsymbol{x}, u, \partial u, \ldots, \partial^{k-1} u\right), \psi^{i j}\left(t, \boldsymbol{x}, u, \partial u, \ldots, \partial^{k-1} u\right)$ with $\psi^{i j}=-\psi^{j i}$, for all solutions $u(t, \boldsymbol{x})$ of PDE (3.1). Only nontrivial conservation laws (3.2) are of interest.

All nontrivial conservation laws (3.2) of PDE (3.1) can be shown to arise from multipliers on the PDE, similarly to Theorem 2.4. We move off the solution space of Eq. (3.1) and let $u(t, \boldsymbol{x})$ be an arbitrary function of $t, \boldsymbol{x}$. We use the notation $\partial_{t}^{q} u=\partial^{q} u / \partial t^{q}$ for pure $t$ derivatives of $u$, and $u_{i}=\partial u / \partial x^{i}, u_{i j}=\partial^{2} u / \partial x^{i} \partial x^{j}$, etc. for pure $\boldsymbol{x}$ derivatives of $u$, and $\partial_{t}^{q} u_{i}=\partial^{q+1} u / \partial t^{q} \partial x^{i}, \partial_{t}^{q} u_{i j}=\partial^{q+2} u / \partial t^{q} \partial x^{i} \partial x^{j}$, etc. for mixed $t, \boldsymbol{x}$ derivatives of $u$, with $\partial_{0}^{q} u=u$ and $\partial_{t}^{0} u_{i}=u_{i}$.

Theorem 3.2 For the Cauchy-Kovalevskaya scalar PDE (3.1), every nontrivial conservation law (3.9) is uniquely characterized by a multiplier $\Lambda$ with no dependence on $\partial_{t}^{N} u$ and differential consequences. The multiplier satisfies the relations

$$
\left(\partial_{t}^{N} u+g\right) \Lambda=D_{t} \Phi^{t}+D_{i}\left(\Phi^{i}-\Gamma^{i}\right)
$$

and

$$
\Lambda=\hat{E}\left(\Phi^{t}\right)
$$

holding for all functions $u(t, \boldsymbol{x})$, where

$$
\hat{E}=\frac{\partial}{\partial\left(\partial_{t}^{N-1} u\right)}-D_{i} \frac{\partial}{\partial\left(\partial_{t}^{N-1} u_{i}\right)}+D_{i} D_{j} \frac{\partial}{\partial\left(\partial_{t}^{N-1} u_{i j}\right)}+\cdots
$$

is a restricted Euler operator, and $\Gamma^{i}$ is given by an expression proportional to $\partial_{t}^{N} u+g$ and its differential consequences.

From Eq. (3.4) one can show that $\Lambda$ is invariant under a change in $\Phi^{t}$ by a trivial conserved density (2.7). (In particular, if $\Phi^{t}$ is trivial, then $\Lambda$ is identically zero, and conversely.) Consequently, it is natural to define the order of a conservation law (3.2) as the order of the highest derivatives of $u$ in its multiplier (3.4).

It is straightforward to derive both the determining system for multipliers $\Lambda$ and the construction formula for conserved densities in terms of $\Lambda$ by applying the results in Sec. 2.1 and 2.2 to the scalar PDE (3.1) written as a first-order Cauchy-Kovalevskaya system (which we carry out later).

In order to display the determining equations explicitly, we introduce the $N+1$ expressions

$$
\begin{aligned}
& \Omega_{0}=\Lambda \\
& \Omega_{q}=(-1)^{q} \mathcal{D}_{t}^{q} \Lambda+\sum_{k=1}^{q}(-1)^{q-k} \mathcal{D}_{t}^{q-k}\left(\frac{\partial g}{\partial\left(\partial_{t}^{N-k} u\right)} \Lambda-D_{i}\left(\frac{\partial g}{\partial\left(\partial_{t}^{N-k} u_{i}\right)} \Lambda\right)+\cdots\right. \\
& \left.\quad+(-1)^{m} D_{i_{1}} \cdots D_{i_{m}}\left(\frac{\partial g}{\partial\left(\partial_{t}^{N-k} u_{i_{1} \cdots i_{m}}\right)} \Lambda\right)\right), q=1, \ldots, N
\end{aligned}
$$


where $\mathcal{D}_{t}$ is the total derivative operator with respect to $t$ on the solution space of the PDE (3.1) as defined by eliminating $\partial_{t}^{N} u=-g$ and all differential consequences. (In particular, $\mathcal{D}_{t} u=\partial_{t} u, \mathcal{D}_{t}^{2} u=\partial_{t}^{2} u$, etc., and $\mathcal{D}_{t}^{N} u=-g$.) Note that, if the order of $\Omega_{0}$ with respect to $\boldsymbol{x}$ derivatives of $u$ is $p$, the order of $\Omega_{q}$ is at most $p+m q$.

Theorem 3.3 For the Cauchy-Kovalevskaya scalar PDE (3.1), the multipliers for all nontrivial conservation laws (3.A) up to any given order $p$ are the solutions $\Lambda\left(t, \boldsymbol{x}, u, \partial u, \ldots, \partial^{p} u\right)$ of the determining system

$$
\Omega_{N}=0
$$

and

$$
\begin{aligned}
& \frac{\partial \Omega_{k}}{\partial\left(\partial_{t}^{j} u\right)}-\frac{\partial \Omega_{j}}{\partial\left(\partial_{t}^{k} u\right)}=\sum_{k=1}^{p^{\prime}}(-1)^{k} D_{i_{1}} \cdots D_{i_{k}} \frac{\partial \Omega_{k}}{\partial\left(\partial_{t}^{j} u_{i_{1} \cdots i_{k}}\right)}, \\
& \frac{\partial \Omega_{k}}{\partial\left(\partial_{t}^{j} u_{i_{1} \cdots i_{q}}\right)}-(-1)^{q} \frac{\partial \Omega_{j}}{\partial\left(\partial_{t}^{k} u_{i_{1} \cdots i_{q}}\right)} \\
& \quad=\sum_{k=q+1}^{p^{\prime}}(-1)^{k-q+1} \frac{k !}{q !(k-q) !} D_{i_{q+1}} \cdots D_{i_{k}} \frac{\partial \Omega_{k}}{\partial\left(\partial_{t}^{j} u_{i_{1} \cdots i_{k}}\right)}, q=1, \ldots, p^{\prime}-1 \\
& \frac{\partial \Omega_{k}}{\partial\left(\partial_{t}^{j} u_{i_{1} \cdots i_{p^{\prime}}}\right)}-(-1)^{p^{\prime}} \frac{\partial \Omega_{j}}{\partial\left(\partial_{t}^{k} u_{i_{1} \cdots i_{p^{\prime}}}\right)}=0,
\end{aligned}
$$

where $p^{\prime}=p+m k, j=0,1, \ldots, N-1 ; k=0,1, \ldots, N-1$.

In this system, Eq. (3.7) is the determining equation for the adjoint symmetries $\Lambda=$ $\omega\left(t, \boldsymbol{x}, u, \partial u, \ldots, \partial^{p} u\right)$ of order $p$ of the PDE (3.1), explicitly

$$
0=\left(-\mathcal{D}_{t}\right)^{N} \omega+\mathcal{L}_{g}^{*} \omega
$$

The extra determining equations (3.8) are the necessary and sufficient conditions for an adjoint symmetry to be a conservation law multiplier. Since Eqs. (3.7) and (3.8) do not involve $\partial_{t}^{N} u$ or any of its differential consequences, one is able to work equivalently on the solution space of the PDE (3.1) in order to find the solutions $\Lambda$.

In order now to display explicitly the construction formula for the conserved densities $\Phi^{t}, \Phi^{i}$ in terms of the multiplier $\Lambda$, we first define the trilinear expression

$$
\begin{aligned}
S^{i}[V, W ; F]= & \sum_{j=0}^{N-1}\left(D_{t}^{j} V\left(\frac{\partial F}{\partial\left(\partial_{t}^{j} u_{i}\right)} W-D_{i_{1}}\left(\frac{\partial F}{\partial\left(\partial_{t}^{j} u_{i i_{1}}\right)} W\right)+\cdots\right)\right. \\
& \left.+D_{t}^{j} D_{j_{1}} V\left(\frac{\partial F}{\partial\left(\partial_{t}^{j} u_{i j_{1}}\right)} W-D_{i_{1}}\left(\frac{\partial F}{\partial\left(\partial_{t}^{j} u_{i i_{1} j_{1}}\right)} W\right)+\cdots\right)+\cdots\right)
\end{aligned}
$$

depending on arbitrary functions $V, W, F$. Next we let

$$
u_{(\lambda)}=\lambda u+(1-\lambda) \tilde{u}
$$

where $\tilde{u}$ is any function of $t, \boldsymbol{x}$. This defines a one-parameter $\lambda$ family of functions with 
$u_{(1)}=u$ and $u_{(0)}=\tilde{u}$. Then we define

$$
\begin{aligned}
& \Omega_{q}\left[u_{(\lambda)}\right]=\Omega_{q}\left(t, \boldsymbol{x}, u_{(\lambda)}, \partial u_{(\lambda)}, \ldots, \partial^{p} u_{(\lambda)}\right), q=0,1, \ldots, N-1 \\
& g\left[u_{(\lambda)}\right]=g\left(t, \boldsymbol{x}, u_{(\lambda)}, \partial u_{(\lambda)}, \ldots, \partial^{p} u_{(\lambda)}\right), \\
& K(t, \boldsymbol{x})=\left(\partial_{t}^{N} \tilde{u}+g[\tilde{u}]\right) \Omega_{0}[\tilde{u}],
\end{aligned}
$$

using Eq. 3.6 for $\Omega_{q}$ in terms of $\Lambda$.

Theorem 3.4 For the Cauchy-Kovalevskaya scalar PDE (3.1), the conserved densities of any nontrivial conservation law (3.9) are given in terms of the multiplier $\Lambda$ by

$$
\begin{aligned}
& \Phi^{t}= \int_{0}^{1} d \lambda \sum_{j=0}^{N-1}\left(\partial_{t}^{j} u-\partial_{t}^{j} \tilde{u}\right) \Omega_{j}\left[u_{(\lambda)}\right]+t \int_{0}^{1} d \lambda K(\lambda t, \lambda \boldsymbol{x}) \\
& \Phi^{i}=x^{i} \int_{0}^{1} d \lambda \lambda^{n} K(\lambda t, \lambda \boldsymbol{x})+\int_{0}^{1} d \lambda\left(S^{i}\left[u-\tilde{u}, \Omega_{0}\left[u_{(\lambda)}\right] ; g\left[u_{(\lambda)}\right]\right]\right. \\
&\left.\quad+S^{i}\left[u-\tilde{u}, g\left[u_{(\lambda)}\right]-\lambda g[u]+(1-\lambda) \partial_{t}^{N} \tilde{u} ; \Omega_{0}\left[u_{(\lambda)}\right]\right]\right) .
\end{aligned}
$$

In applying the construction formula 3.15$)$ and $(3.16)$, we fix the function $\tilde{u}$ so that the expressions $\Lambda[\tilde{u}]$ and $g[\tilde{u}]$ are nonsingular. In particular, if $\Lambda[0]$ and $g[0]$ are nonsingular then we can choose $\tilde{u}=0$, which significantly simplifies the integrals. Moreover, if $\tilde{u}=$ $u=0$ satisfies the PDE (3.1), then immediately the $K$ integrals vanish. A change in the choice of $\tilde{u}$ alters the conserved densities only by a trivial conserved density (2.7).

\section{Conversion to a first order Cauchy-Kovalevskaya system:}

We now outline the proof of Theorems 3.3 and 3.4 using Theorems 2.6 and 2.8. To begin we write the scalar PDE (3.1) in first-order (evolution) form (1.1) with respect to $t$ as follows:

$$
\begin{aligned}
& u^{1}=u, u^{2}=\partial_{t} u, \ldots, u^{N}=\partial_{t}^{N-1} u \\
& g^{1}=-u^{2}, \ldots, g^{N-1}=-u^{N}, g^{N}=g \\
& G^{1}=\partial_{t} u^{1}-u^{2}=0, \ldots, G^{N-1}=\partial_{t} u^{N-1}-u^{N}=0, G^{N}=\partial_{t} u^{N}+g=0 .
\end{aligned}
$$

Through Eqs. (3.17) to (3.19) there is a one-to-one correspondence between nontrivial conservation laws (3.2) of the scalar PDE (3.1) and nontrivial conservation laws in normal form (2.8) of the equivalent first-order PDE system (3.19). The relation between a multiplier $\Lambda$ of a scalar PDE conservation law and a set of multipliers $\left\{\Lambda_{1}, \ldots, \Lambda_{N}\right\}$ of the corresponding PDE system conservation law can be obtained by considering the adjoint symmetry equations of the scalar PDE (3.1) and the PDE system (3.19). Straightforwardly, from Eqs. (3.18) and 2.22), we have

$$
\begin{aligned}
& 0=-\mathcal{D}_{t} \Lambda_{N}+\mathcal{L}_{0, g}^{*} \Lambda_{1}, \\
& 0=-\mathcal{D}_{t} \Lambda_{N-q}-\Lambda_{N-q+1}+\mathcal{L}_{q, g}^{*} \Lambda_{1}, q=1, \ldots, N-1
\end{aligned}
$$


where $\mathcal{L}_{q, g}^{*}$ is the adjoint operator of the linearization operator $\mathcal{L}_{q, g}$ defined by

$$
\mathcal{L}_{q, g}=\frac{\partial g}{\partial\left(\partial_{t}^{q} u\right)}+\frac{\partial g}{\partial\left(\partial_{t}^{q} u_{i}\right)} D_{i}+\cdots+\frac{\partial g}{\partial\left(\partial_{t}^{q} u_{i_{1} \cdots i_{m}}\right)} D_{i_{1}} \cdots D_{i_{m}}
$$

By solving Eq. 3.21) for $\Lambda_{2}, \ldots, \Lambda_{N}$ in terms of $\Lambda_{1}$ and comparing Eq. (3.20) with Eq. (3.7), we directly see

$$
\Lambda_{1}=\Lambda=\Omega_{0}, \Lambda_{2}=\Omega_{1}, \ldots, \Lambda_{N}=\Omega_{N-1} .
$$

This establishes an explicit correspondence between $\Lambda$ and $\left\{\Lambda_{1}, \ldots, \Lambda_{N}\right\}$ leading immediately to Theorems 3.3 and 3.4 from Theorems 2.6 and 2.8 .

Remarks on the determining system and construction formula:

Theorems 3.3 and 3.4 can also be established directly from Theorem 3.2 without use of the results in Sec. 2.1 and 2.2. The main step in the proof of Theorem 3.3 is a polynomial splitting result analogous to Lemma 2.5 as follows.

The determining condition for a multiplier $\Lambda$ of order $p$ for the scalar PDE (3.1) arises from the relation (3.3) by the result that an expression is a divergence if and only if it is annihilated by the full Euler operator

$$
E_{u}=\frac{\partial}{\partial u}-D_{i} \frac{\partial}{\partial u_{i}}-D_{t} \frac{\partial}{\partial\left(\partial_{t} u\right)}+D_{i} D_{j} \frac{\partial}{\partial u_{i j}}+D_{t} D_{j} \frac{\partial}{\partial\left(\partial_{t} u_{j}\right)}+D_{t}^{2} \frac{\partial}{\partial\left(\partial_{t}^{2} u\right)}+\cdots
$$

This can be shown (by a straightforward calculation [11]) to yield

$$
0=E_{u}\left(\left(\partial_{t}^{N} u\right) \Lambda+g \Lambda\right)=\left(-D_{t}\right)^{N} \Lambda+\mathcal{L}_{g}^{*} \Lambda+\mathcal{L}_{\Lambda}^{*}\left(\partial_{t}^{N} u+g\right),
$$

which is required to hold for all functions $u(t, \boldsymbol{x})$ (not just solutions of Eq. (3.1)). The determining condition (3.25) is a polynomial in $\partial_{t}^{N} u, \partial_{t}^{N+1} u, \ldots, \partial_{t}^{2 N-1} u$ and differential consequences with respect to $\boldsymbol{x}$. Furthermore, the terms in this polynomial have weights 0 up to $N$, where we assign weight 1 to $\partial_{t}^{N} u$ (and $\boldsymbol{x}$ derivatives of $\partial_{t}^{N} u$ ), 2 to $\partial_{t}^{N+1} u$ (and $\boldsymbol{x}$ derivatives of $\partial_{t}^{N+1} u$ ), etc., and we add the weights of products (and powers) of $\partial_{t}^{N} u, \partial_{t}^{N+1} u$, etc.. Now, since $u$ is required to be an arbitrary function of $t$ and $\boldsymbol{x}$, the polynomial splits into separate determining equations given by the coefficients of the various weight terms involving $\partial_{t}^{N} u, \partial_{t}^{N+1} u, \ldots, \partial_{t}^{2 N-1} u$ (and differential consequences with respect to $\boldsymbol{x}$ ). It is convenient to organize the splitting by working in terms of $\partial_{t}^{N} u+g=G, \partial_{t}^{N+1} u+\mathcal{D}_{t} g=\mathcal{D}_{t} G, \partial_{t}^{N} u_{i}+D_{i} g=D_{i} G, \partial_{t}^{N+1} u_{i}+D_{i} \mathcal{D}_{t} g=D_{i} \mathcal{D}_{t} G$, etc.. The terms of weight 0 yield the adjoint symmetry determining equation (3.7) and the terms of weight 1 up to $N$ yield the extra determining equations (3.8) on $\Lambda$. This derivation is illustrated in the second example of Part I.

The construction formula for conserved densities $\Phi^{t}$ and $\Phi^{i}$ of a conservation law for PDE (3.1) is obtained by inverting the Euler operator equation (3.25) as follows. Since Eq. (3.25) holds for arbitrary functions $u(t, \boldsymbol{x})$, it must hold with $u$ replaced by the one-parameter family $u_{(\lambda)}=\lambda u+(1-\lambda) \tilde{u}$. This yields

$$
0=\left(-D_{t}\right)^{N} \Lambda\left[u_{(\lambda)}\right]+\mathcal{L}_{g\left[u_{(\lambda)}\right]}^{*} \Lambda\left[u_{(\lambda)}\right]+\mathcal{L}_{\Lambda\left[u_{(\lambda)}\right]}^{*}\left(\partial_{t}^{N} u_{(\lambda)}+g\left[u_{(\lambda)}\right]\right) .
$$

We multiply Eq. (3.26) by $u-\tilde{u}$ and then rearrange the terms which involve total 
derivative operators coming from $\mathcal{L}_{g}^{*}$ and $\mathcal{L}_{\Lambda}^{*}$. This leads to the formula

$$
\begin{aligned}
& D_{t}\left(\sum_{j=0}^{N-1}\left(\partial_{t}^{j} u-\partial_{t}^{j} \tilde{u}\right) \Omega_{j}\left[u_{(\lambda)}\right]\right)+D_{i}\left(S^{i}\left[u-\tilde{u}, \Omega_{0}\left[u_{(\lambda)}\right] ; g\left[u_{(\lambda)}\right]\right]\right. \\
& \left.\quad+S^{i}\left[u-\tilde{u}, \partial_{t}^{N} u_{(\lambda)}+g\left[u_{(\lambda)}\right] ; \Omega_{0}\left[u_{(\lambda)}\right]\right]\right)=\frac{\partial}{\partial \lambda}\left(\left(\partial_{t}^{N} u_{(\lambda)}+g\left[u_{(\lambda)}\right]\right) \Lambda\left[u_{(\lambda)}(3) .\right) 27\right)
\end{aligned}
$$

Next we integrate from $\lambda=0$ to $\lambda=1$ and apply the fundamental theorem of calculus. Using the identity $D_{t}\left(t \int_{0}^{1} d \lambda K(\lambda t, \lambda \boldsymbol{x})\right)+D_{i}\left(x^{i} \int_{0}^{1} d \lambda \lambda^{n} K(\lambda t, \lambda \boldsymbol{x})\right)=K$, and finally moving onto the solution space of the PDE (3.1), we obtain the conservation law (3.2) with $\Phi^{t}$ and $\Phi^{i}$ given by Eqs. (3.15) and (3.16).

\section{Remarks on variational principles:}

Definition 3.5 A Cauchy-Kovalevskaya scalar PDE (3.1) is called variational if it arises from an action

$$
S=\int\left(L\left(t, \boldsymbol{x}, u, \partial u, \ldots, \partial^{k} u\right)\right) d t d \boldsymbol{x}
$$

by variation with respect to $u$,

$$
G=E_{u}(L)=\partial_{t}^{N} u+g .
$$

The well-known necessary and sufficient condition 11] for existence of an action (3.28) is that

$$
D_{t}^{N}+\mathcal{L}_{g}=\left(-D_{t}\right)^{N}+\mathcal{L}_{g}^{*}
$$

i.e. $N$ must be even and $g$ must have a self-adjoint linearization. This condition is equivalent to requiring that the determining equation for symmetries of the PDE (3.1) is self-adjoint.

In the case when PDE (3.1) is variational, Theorem 3.3 combined with Noether's theorem [5, 11] shows that the extra determining equations (3.8) constitute necessary and sufficient conditions for a symmetry of the PDE (3.1) to leave invariant the action (3.28) to within a boundary term. In particular, if $\mathrm{X} u=\eta\left(t, \boldsymbol{x}, u, \partial u, \ldots, \partial^{p} u\right)$ is a symmetry of order $p$, then $\mathrm{X} S=\int\left(D_{t} \theta^{t}+D_{i} \theta^{i}\right) d t d \boldsymbol{x}$ holds for some expressions $\theta^{t}$ and $\theta^{i}$ iff $\Lambda=\eta$ satisfies Eq. (3.8) and hence $\eta$ is a multiplier yielding a conservation law (3.2) of PDE (3.1).

\section{Summary and concluding remarks}

For any Cauchy-Kovalevskaya system $G$ of one or more PDEs, Theorems 2.6, 2.8 and Theorems 3.3, 3.4 yield an effective computational method to obtain all local conservation laws (up to any specified order). The method is summarized as follows:

1. Linearize $G$ to form its linearized system $\ell$, which is the determining system for the symmetries of $G$.

2. Form the adjoint system $\ell^{*}$ of $\ell$, which is the determining system for the adjoint symmetries of $G$. 
3. Form the extra system $h$ comprising the necessary and sufficient determining equations for an adjoint symmetry to be a multiplier for a conservation law of $G$.

4. Solve the augmented system $\ell^{*} \cup h$. This is the determining system for the multipliers that yield all nontrivial local conservation laws of $G$.

5. Use the explicit construction formula to obtain the conserved densities arising for each solution of the system $\ell^{*} \cup h$.

The linearized system of $G$ is self-adjoint $\left(\ell=\ell^{*}\right)$ if and only if $G$ is variational, in which case solutions of $\ell^{*}$ are solutions of $\ell$. Then the extra system $h$ is equivalent to the condition for symmetries to leave invariant the action for $G$. In general, if $G$ is not variational then solutions of $\ell^{*}$ are not solutions of $\ell$.

The systems $\ell, \ell^{*}, h$, and $\ell^{*} \cup h$ are all linear overdetermined systems which are solved working entirely on the space of solutions of $G$ (i.e. a leading derivative of the dependent variables in $G$ is eliminated). There exist algorithmic procedures [9] to seek solutions of $\ell$. These procedures can be readily adapted for seeking solutions of $\ell^{*}, h$, and $\ell^{*} \cup h$. In general, $\ell^{*} \cup h$ is more overdetermined than $\ell$ and hence is typically easier to solve. More significantly, one can choose appropriate mixings of the determining equations in $\ell^{*}$ and $h$ to solve $\ell^{*} \cup h$ effectively.

One can also use specific ansatze to seek particular solutions of $\ell^{*} \cup h$, such as restricting the form of highest derivatives of the dependent variables of $G$ allowed in the solution. For example, familiar conservation laws such as energy invariably arise from the simple ansatz of seeking multipliers restricted to be linear in first derivatives.

In general it is important to note that solutions of $\ell^{*}$ are not necessarily solutions of $h$ and hence $\ell^{*}$ does not determine a conservation law multiplier. This typically occurs for scaling symmetries of systems $G$ in the case $\ell^{*}=\ell$ (i.e. self-adjoint), and for pointtype adjoint symmetries (first-order and linear in derivatives of dependent variables) of systems $G$ in the case $\ell^{*}=-\ell$ (i.e. skew-adjoint). Examples are $u_{t t}-u_{x x}+u^{3}=0$ which has $u+t u_{t}+x u_{x}$ as a solution of $\ell=\ell^{*}$ but not a solution of $h ; u_{t}+u_{x x x}=0$ which has $u_{x}$ as a solution of $\ell^{*}=-\ell$ but not a solution of $h$. Ref. [2] exhibits several ODE examples in which nontrivial adjoint symmetries are not multipliers. The need for the extra conditions $h$ to determine multipliers has not been clearly recognized in the literature (e.g. [6]).

The chief aspect of our method compared to other existing treatments of PDE conservation laws (e.g. [7, 13, 11, [5, 14]) is the explicit delineation of the linear determining system $\ell^{*} \cup h$ which incorporates (and identifies) the necessary and sufficient conditions for adjoint symmetries to be multipliers, without moving off the space of solutions of the given $\mathrm{PDE}(\mathrm{s}) G$. Consequently, one can calculate multipliers of conservation laws by effective algorithmic procedures. Moreover there is the added computational advantage of allowing the determining equations in the adjoint system $\ell^{*}$ and the extra system $h$ to be mingled to optimally solve the determining system $\ell^{*} \cup h$, as illustrated by the conservation law classification results for the PDE examples in Part I. 


\section{Acknowledgements}

The authors are supported in part by the Natural Sciences and Engineering Research Council of Canada. We gratefully thank the referees for useful comments which have improved this paper.

\section{References}

[1] S.C. Anco and G. Bluman, Direct construction of conservation laws from field equations, Phys. Rev. Lett. 78, 2869-2873 (1997).

[2] S.C. Anco and G. Bluman, Integrating factors and first integrals of ordinary differential equations, Eur. J. Appl. Math. 9, 245-259 (1998).

[3] S.C. Anco and G. Bluman, Direct construction method for conservation laws of partial differential equations. Part I: Examples of conservation law classifications. Eur. J. Appl. Math. 13, 545-566 (2002).

[4] T.B. Benjamin, J.L. Bona, and J.J. Mahoney, Model equations for long waves in nonlinear dispersive media, Phil. Trans. Roy. Soc. A272, 47-48 (1972).

[5] G. Bluman and S. Kumei, Symmetries and Differential Equations (Springer, New York 1989).

[6] N. Chien, T. Honein, G. Herrmann, Dissipative systems, conservation laws and symmetries, Int. J. Solids Structures 33, 2959-2968 (1996).

[7] A.S. Fokas and B. Fuchssteiner, On the structure of symplectic operators and hereditary symmetries, Lett. Al Nuovo Cim. 28, 299-303 (1980).

[8] B. Fuchssteiner and A.S. Fokas, Symplectic structures, their Backlund transformations and hereditary symmetries, Physica 4D, 47-66 (1981).

[9] W. Hereman, CRC Handbook of Lie Group Analysis of Differential Equations, Volume 3: New Trends in Theoretical Developments and Computational Methods. Chapter 13, 367413. (N.H. Ibragimov, ed.) (CRC Press, Boca Raton, Florida 1996).

[10] B.B. Kadomtsev and V.I. Petviashvili, On the stability of solitary waves in weakly dispersive media, Sov. Phys. Dokl. vol15, 539-541 (1970).

[11] P.J. Olver, Applications of Lie Groups to Differential Equations (Springer, New York, 1986).

[12] C.E. Seyler and D.L. Fenstermacher, A symmetric regularized long wave equation, Phys. Fluids 27, 4-7 (1984).

[13] A.M. Vinogradov, Local symmetries and conservation laws, Acta. Appl. Math. 2, 21-78 (1984).

[14] T. Wolf, A comparison of four approaches to the calculation of conservation laws. Eur. J. Appl. Math. 13, 129-152 (2001). 